\title{
Super-resolution Photonic Nanojet Interferometry: Photonic Nanojet Interaction with a Polymer Sample
}

\author{
Maria Gritsevich $^{\mathrm{a}, \mathrm{b}}$, Göran Maconi ${ }^{\mathrm{a}}$, Anton Nolvi ${ }^{\mathrm{a}, \mathrm{c}}$, Ivan Kassamakov ${ }^{\mathrm{a}}$, Antti Penttiläa ${ }^{\mathrm{a}}$, Karri Mui- \\ nonen $^{\mathrm{a}, \mathrm{d}}$, and Edward Hæggström ${ }^{\mathrm{a}}$ \\ a'Department of Physics, Gustaf Hällströmin katu 2a, P.O. Box 64, FI-00014 U. Helsinki, Finland \\ bInstitute of Physics and Technology, Ural Federal University, 620002 Ekaterinburg, Russia \\ cÅbo Akademi, Turku, Finland \\ ${ }^{\mathrm{d}}$ Finnish Geospatial Research Institute FGI, Geodeetinrinne 2, FI-02430 Masala, Finland
}

email: ivan.kassamakov@helsinki.fi

\begin{abstract}
Super-resolution photonic nanojet interferometry is a new modality for 3D label-free super-resolution imaging. We present a comparative study of the photonic nanojet interaction with a polymer sample. We use numerical modelling to understand the interaction between a microsphere-induced photonic nanojet and the sample. The numerical model employs the same set of input parameters (melamine formaldehyde microsphere with a diameter of $11 \mu \mathrm{m}$ and a refractive index of 1.68), as in our experiments. The interaction is described using the Finite-Difference Time-Domain method applied on a tight mesh. The knowledge gained using the verified and validated model will be used to conduct numerical simulations across a wider parameter space. This enables optimizing the design of 3D-interferometric super-resolution microscopes.
\end{abstract}

Keywords: label-free imaging, super-resolution microscopy

\section{INTRODUCTION}

Technological development towards compact and effective devices requires increasingly smaller structures. Complex structures, common in modern sensors and transistors, are produced with sizes of a few tens of nanometers [1]. Furthermore, interest in observations at the nanometer scale is important in both biology and medicine, where understanding the behavior of the components of cells and viruses is critical. The necessity to observe such small details fuels the need for new imaging methods.

Conventional optical microscopy has been the basic tool for resolving small details. Optical microscopes are easy to use, affordable, and provide easily understandable visualization of small structures. The resolution of optical microscopes is limited due to diffraction of light. When using Rayleigh criteria for resolution, this limit is $0.61 \lambda / A$ laterally and $0.61 \lambda / A^{2}$ axially, where $\lambda$ is center wavelength of illumination, and $A$ is the numerical aperture of system [2].

Combining conventional optical microscopy with interferometric techniques, e.g. Scanning White Light Interferometry (SWLI), sub-nanometer height differences in the sample topology can be resolved [3]. The lateral resolution remains at a few hundred nanometers at best. However, a new phenomenon, the photonic nanojet (PNJ), has the ability to focus light beyond the lateral diffraction limit [4].

We studied the combination of PNJ with SWLI by using theoretical simulations and experimental measurements to create a microscope that can resolve sample features at the nanometer scale in 3D. The setup features a single objective and maintains the main features of conventional optical microscope: ease-of-use, affordability, and non-destructive imaging.

ivan.kassamakov@helsinki.fi 


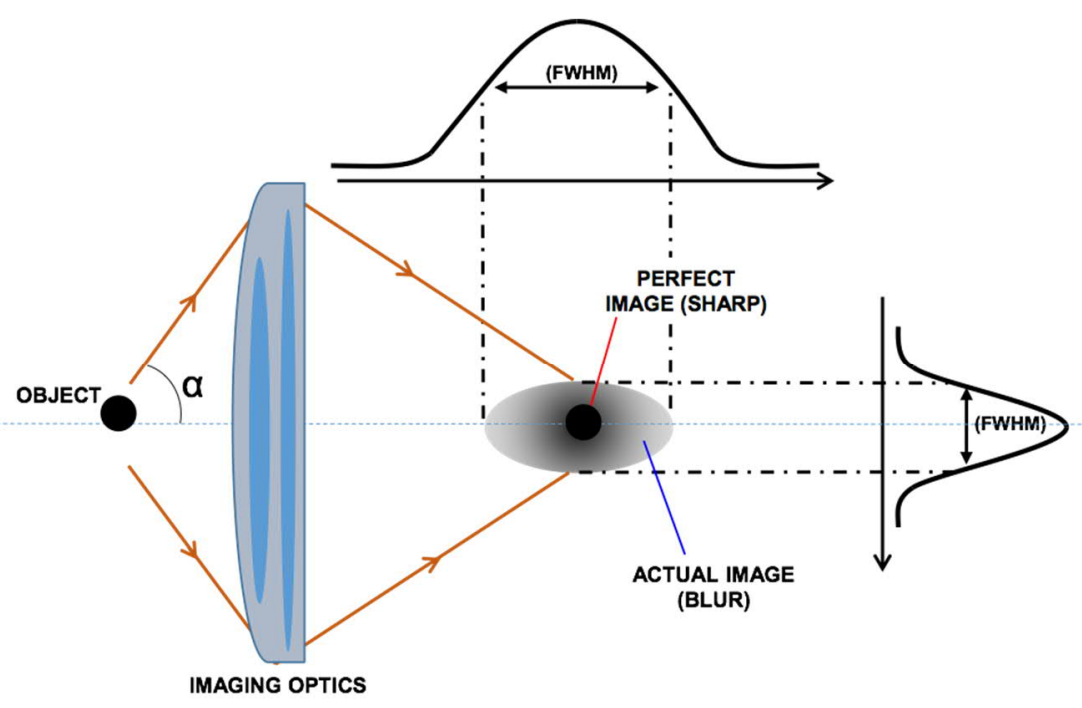

Figure 1. Image formation in a conventional, far-field microscope.

\section{METHODS AND MATERIALS}

Numerical simulations of the photonic nanojet (PNJ) were performed using the Finite-Difference Time-Domain (FDTD) method applied on a finely discretized mesh (see, e.g., [5]). The software was Optiwave OptiFDTD, an integrated application that allows computer-aided design and simulation of linear and nonlinear photonic components. The algorithm solves electric and magnetic fields in temporal and spatial domain using the full-vector differential form of Maxwell's coupled curl equations. Simulations were done in two stages. First, the general shape of the PNJ was simulated using white light, to give the focal distance and width of the generated jet. These parameters were then used in the second stage to simulate the imaging properties of the microsphere in an interferometric setup.

The numerical model employs the same set of input parameters (melamine formaldehyde sphere with a diameter of 11 $\mu \mathrm{m}$ and a refractive index of 1.68), as in our experiments, Fig. 2. The model comprises a plane wave source (with a Gaussian distribution of intensity (full width at $1 / \mathrm{e}^{2}=10 \mu \mathrm{m}$ ) around the axis of symmetry of the simulation), and a microsphere lens.

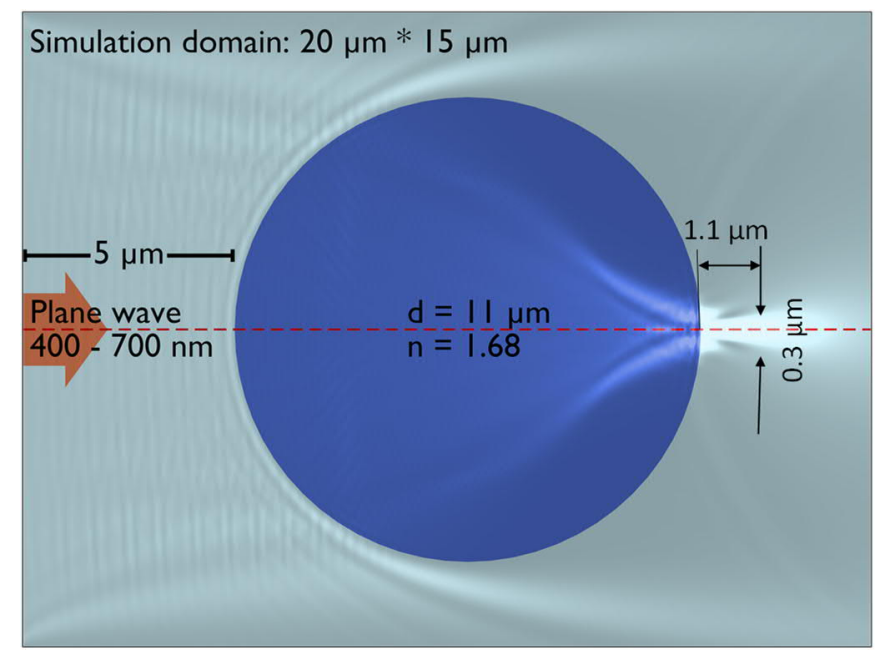

Figure 2. Graphical diagram of the PNJ simulation. 
White light was approximated by a set of simulations that ran at discrete wavelengths ranging from $400 \mathrm{~nm}-700 \mathrm{~nm}$ in $10 \mathrm{~nm}$ increments. This was implemented in the calculations by performing a parameter sweep across the wavelength of the plane wave source. These results were then combined in MATLAB by adding the intensities.

The spatial parameters of the photonic nanojet were identified by finding the focal point, defined as the intensity maximum along the central axis of the simulation domain (Fig. 3a). The beam profile (Fig. 3b) represents a cross-section of the PNJ at its maximum intensity.

a)

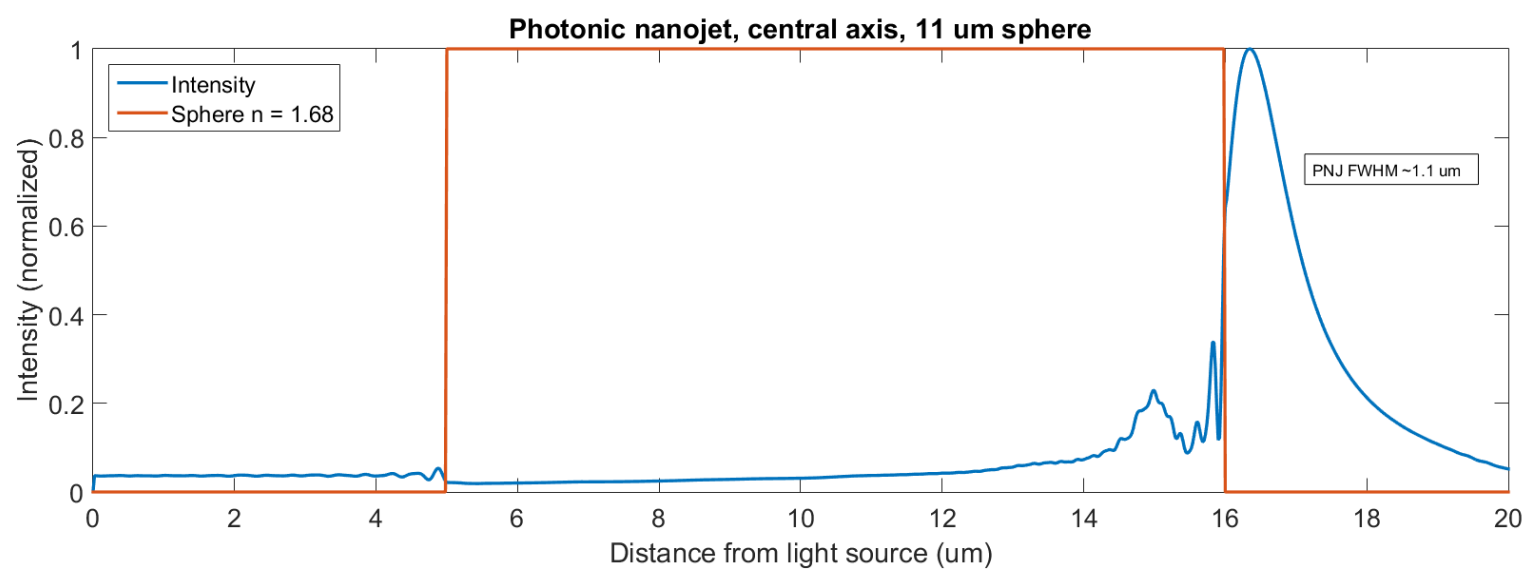

b)

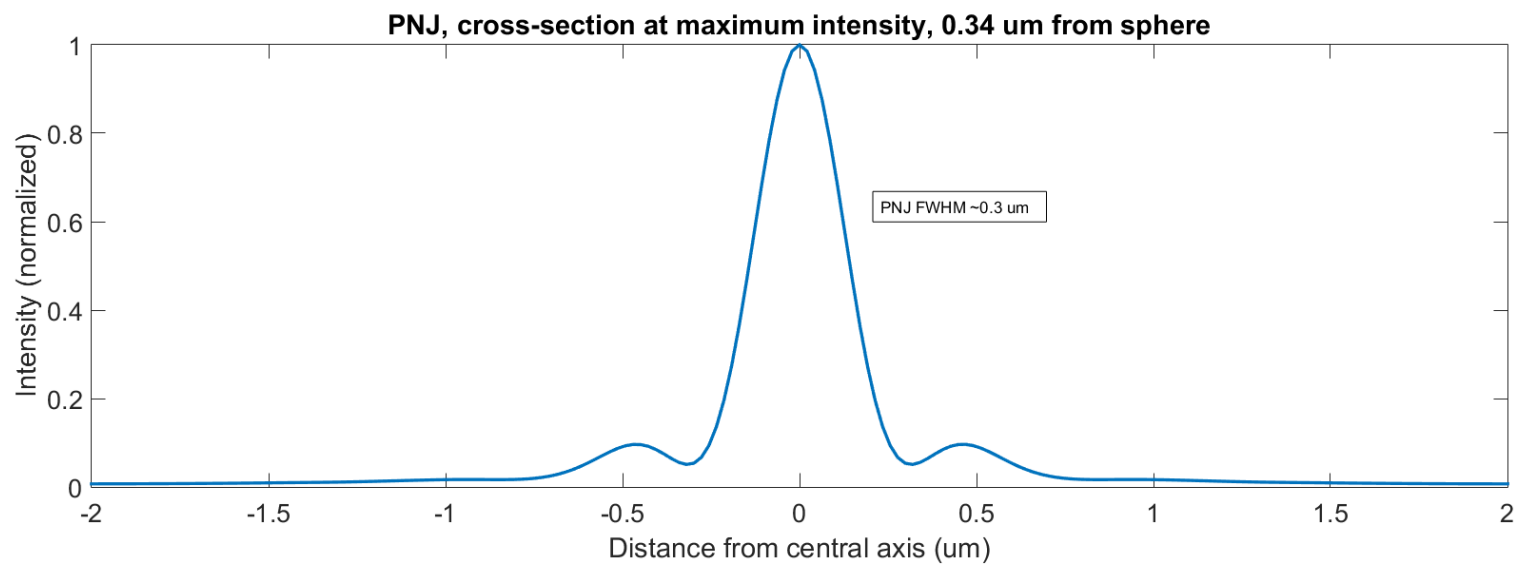

Figure 3. PNJ intensity profile a) along the optical axis, b) across the focal point of the PNJ. The focus is $0.3 \mu \mathrm{m}$ wide (FWHM) and $1.1 \mu \mathrm{m}$ deep.

A Michelson interferometer, with the microsphere acting as an objective lens, was modeled during the second stage of the simulations. The interferometer comprises a beam splitter that divides the incoming light into two paths, one of which is reflected in a planar mirror (reference beam) while the other beam enters the objective. The reflected beams are recombined and focused using a plano-convex lens, Fig. 4.

For simplicity, the object was placed at the focal point of the PNJ, to produce a single-point measurement rather than an imaging plane. Another simplification was to use monochromatic coherent light $(600 \mathrm{~nm})$ in the interferometer, to eliminate the need for the reference beam to have the same optical path length as the sample beam. This allows the size of the simulation domain to be significantly reduced. 


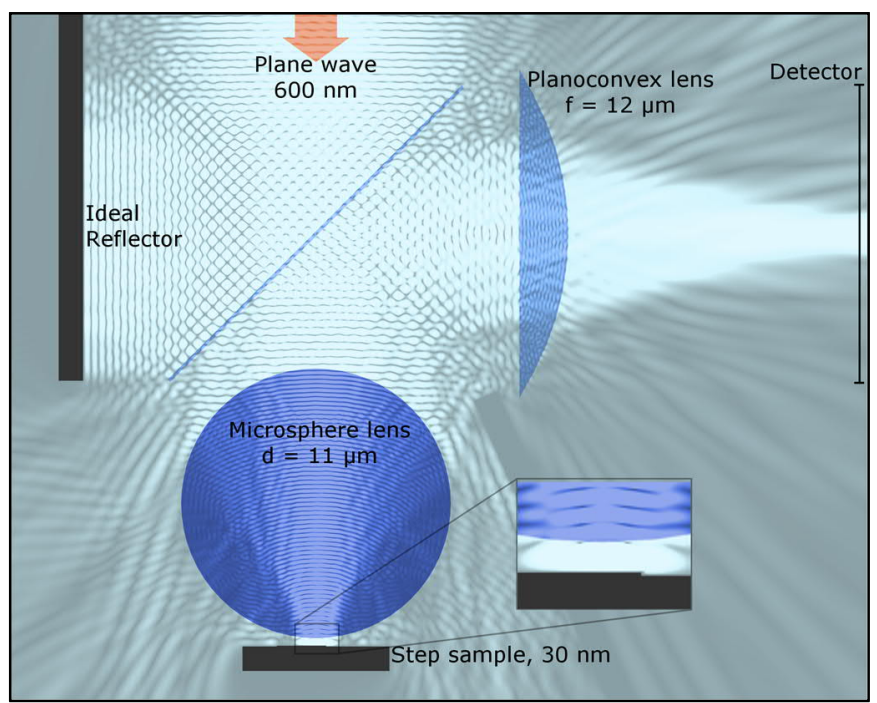

Figure 4. Graphical diagram of the simulated interferometer setup.

In the experiments we imaged the topology of BluRay® disc (BD-R) (Verbatim BD-R Datalife 25GB 6x, BD-RSL:CMCMAG-BA5-000). The sample was selected due to its availability as well as its controlled nanometer-scale topography. The BD-R disc features parallel grooves (Fig. 5) that the laser of the optical disc drive tracks. The grooves are nominally pitched at $320 \mathrm{~nm}$ and feature heights of few tens of nanometers [6]. This topology falls beyond the diffraction limit and cannot be seen with a conventional optical microscope.

The BD-R discs feature protective polymer layers of up to a mm thick [7]. These layers can be manually peeled off to expose the grooves.

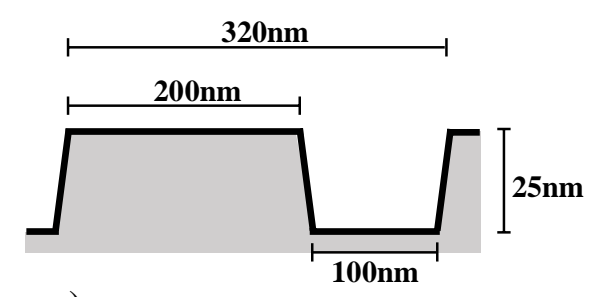

a)

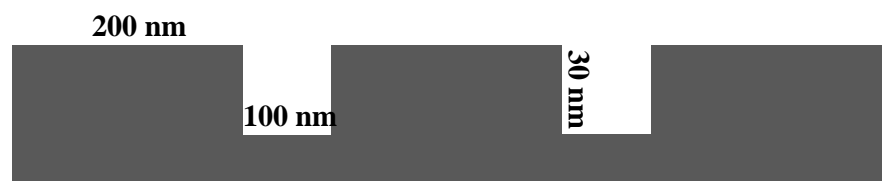

b)

Figure 5. a) Dimensions of a grooved structure of the BD-R disc according to the specification. b) Object geometry used in the simulations.

\section{MEASUREMENT SETUP}

The imaging setup is a SWLI combined with PNJ enhanced objective. The system is built on an infinity corrected Nikon microscope frame (L-UEPI EPI) featuring a 100W halogen light source (Philips 7724). To acquire 3D information of the sample the interference and focus plane is scanned through the sample topography with a PI piezo actuator (Pifoc P721.CDQ) by moving the objective assembly. The system records the images as a function of sample-objective distance with a Hamamatsu CMOS camera (Orca Flash 2.8) focused on the sensor with a Nikon 200mm tube lens of 1x magnification, Fig. 6. From the recorded data, the interference signal can be analyzed as the light intensity is related to the sample-objective distance seen by each pixel [8]. A 3D matrix showing the sample topography can therefore be calculated [9]. 


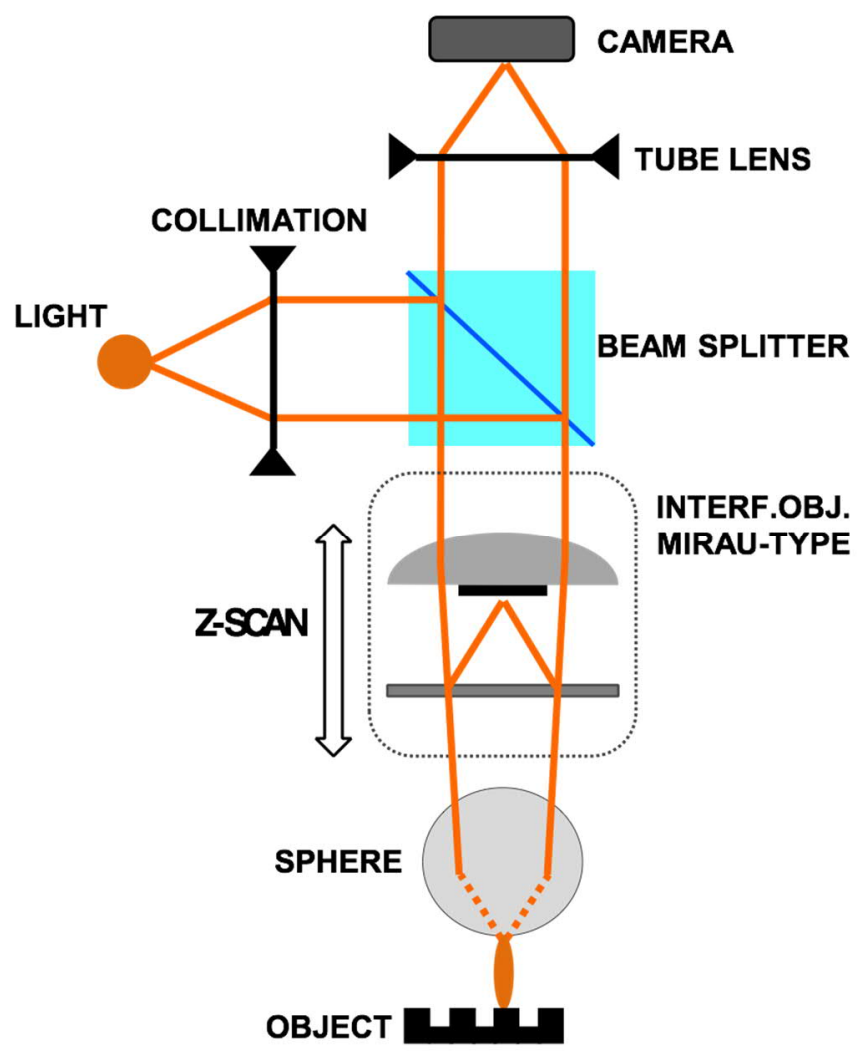

Figure 6. Optical scheme of the setup.

The actual interference image is formed within a Mirau-type interferometric objective. We used a Nikon objective with 50x magnification (CF IC EPI Plan DI 50x, NA=0.55). The objective forms an image of the sample through a PNJcreating microsphere $(\varnothing=11 \mu \mathrm{m})$ made of melamine formaldehyde (Corpuscular, C-MFs-10.0).

In this experiment, we applied the spheres, contained in liquid solution, directly on top of the exposed grating of the BD$\mathrm{R}$ disc. The spheres self-assembled on top of the sample while the liquid evaporated which resulted in multiple individually working PNJ-creating objects. When imaged through this layer, each sphere acted as an additional lens providing additional optical magnification and spherical aberration. Knowing the nominal pitch of the grooves and assuming spherical deformation, the 3D data were corrected with post-processing techniques using a commercial 3D data analysis software, Mountains Map from Digital Surf. The same software was used in all 3D data analysis.

\section{RESULTS}

\subsection{Simulation results}

To demonstrate the resolution of the PNJ, a sample consisting of a perfectly reflective $30 \mathrm{~nm}$ step was scanned laterally, over a range of $400 \mathrm{~nm}$ on each side of the step. The raised plateau of the sample results in positive interference. Figure 7 shows the sharpness of the transition from the base of the sample to the plateau. Across a lateral distance of $100 \mathrm{~nm}$, as in the grating sample, the output shows a difference in intensity of $\sim 20 \%$.

The ability to detect individual features was tested by scanning the PNJ across a rectangular protruding feature (100 nm wide, $30 \mathrm{~nm}$ high) as well as across an equivalent recessed feature, Fig. 8. In the simulations, the protruding feature produced a difference in intensity of $\sim 40 \%$, while the recessed feature only produced a difference of $7 \%$. 

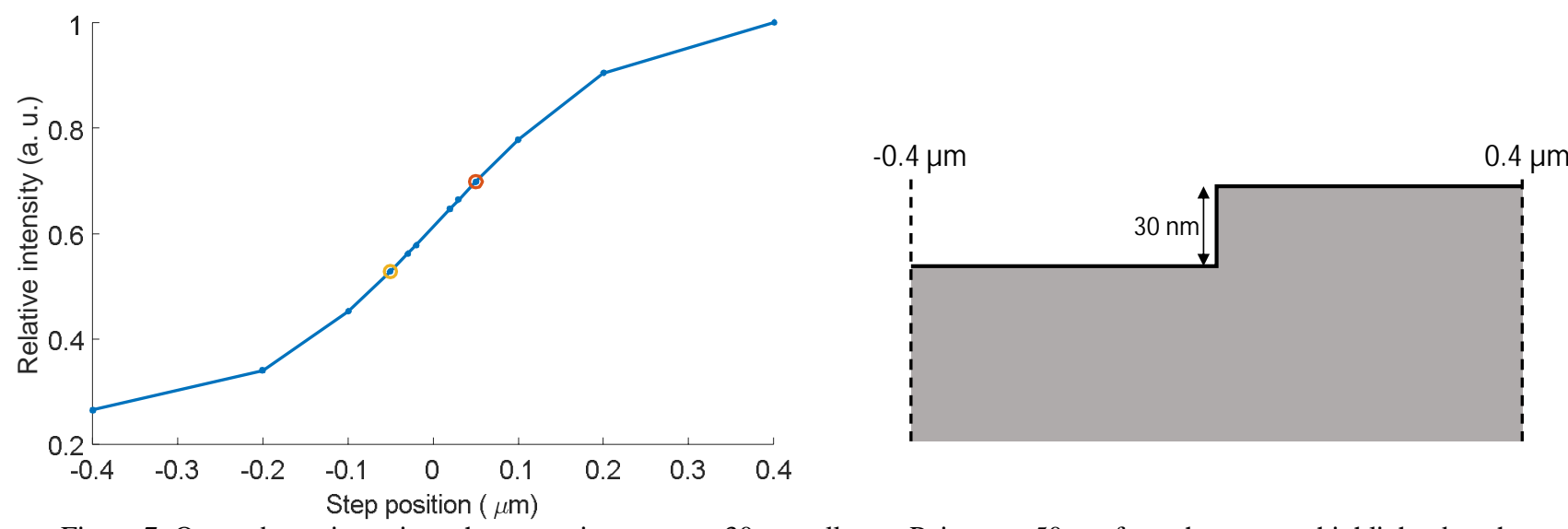

Figure 7. Output beam intensity, when scanning across a $30 \mathrm{~nm}$ tall step. Points at $\pm 50 \mathrm{~nm}$ from the step are highlighted to show the contrast across $100 \mathrm{~nm}$ lateral distance.
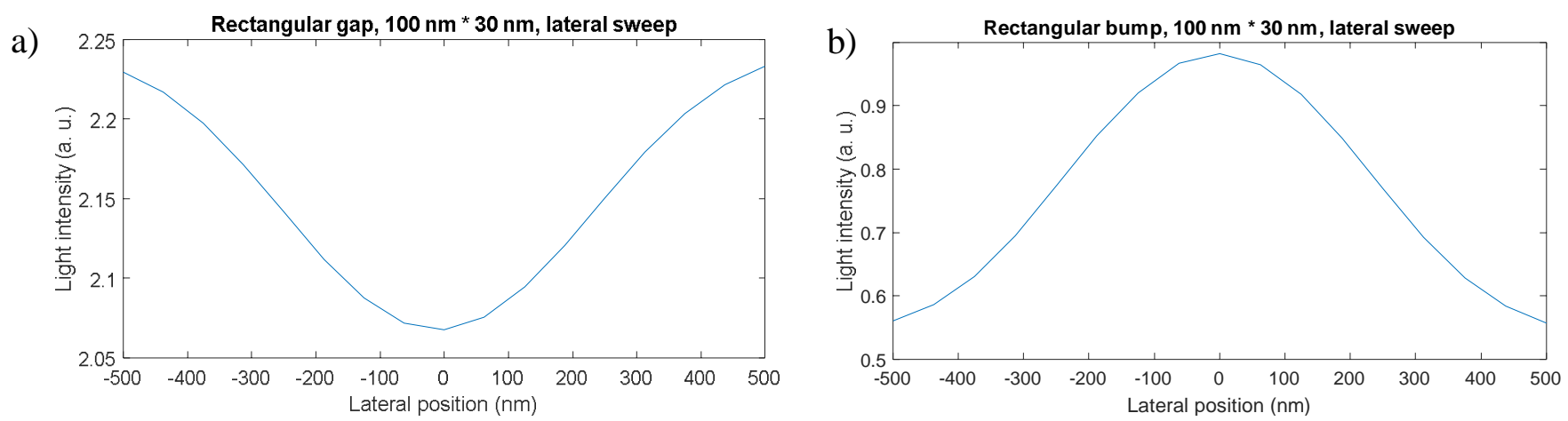

Figure 8. Lateral scan of a) a protruding bump b) a recessed gap, $100 \mathrm{~nm}$ wide. The bump produces a higher relative contrast (40\%) than the gap (7\%).

\subsection{Measurement results}

The measurement results obtained using the described setup and the BluRay® disc are shown in Fig. 9. The surface features which are $100 \mathrm{~nm}$ wide and $25 \mathrm{~nm}$ tall are measurable using the microsphere assisted SWLI
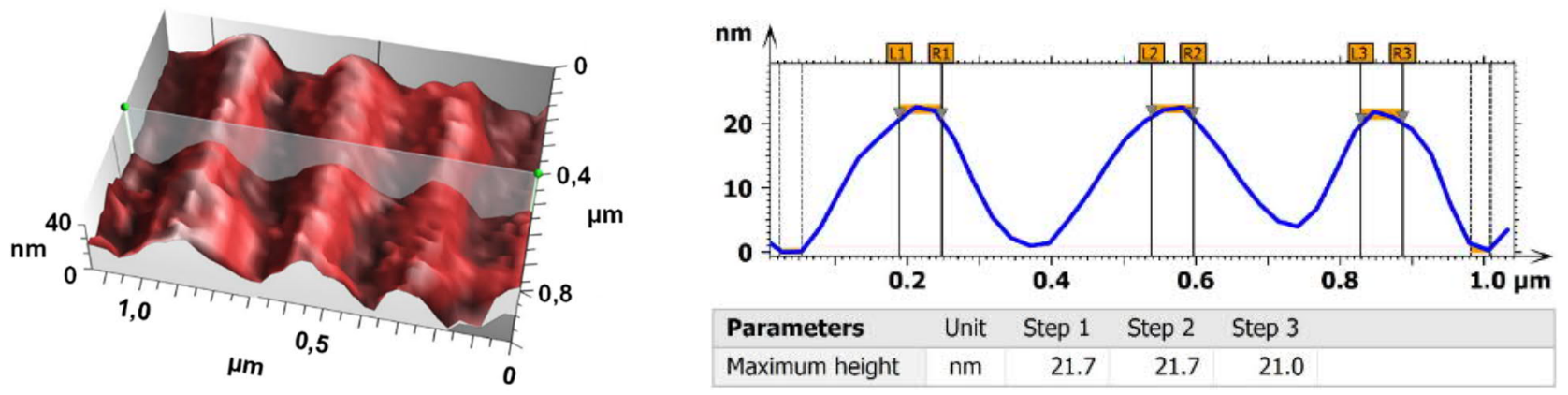

Figure 9. Measurements results of a BluRay® disc. 


\section{DISCUSSION}

Our model shows that an 11- $\mu$ m melamine formaldehyde sphere condenses white light into a PNJ, which makes the sphere a potential key component in a super-resolution interferometric microscope.

Furthermore, in our experiments we demonstrate the ability of the PNJ to resolve details smaller than the diffraction limit. The modeled setup is unable to distinguish the gaps in a grating similar to that found in BluRay® discs. Due to the size limitations of the simulation domain, the far-field behavior of the light reflected from the sample could not be accurately modeled. This simplification of the model restricts us to point scans. For more complex imaging, near-field to farfield calculations are needed.

\section{CONCLUSIONS}

Simulations confirm our ability to resolve features beyond the diffraction limit, using microspheres. The model applied in this study was unable to recreate the far-field imaging plane. A more accurate model including near-field to far-field calculations is a subject of further investigations.

\section{ACKNOWLEDGEMENTS}

This study was conducted under the support from the European Research Council, in the frame of the Advanced Grant project No. 320773 'Scattering and Absorption of Electromagnetic Waves in Particulate Media' (SAEMPL).

\section{REFERENCES}

1. “Intel's 14 nm Technology: Delivering Ultrafast, Energy-Sipping Products," Intel news fact sheet, Intel Corporation 2017, https://newsroom.intel.com/newsroom/wp-content/uploads/sites/11/2017/03/14-nm-technologyfact-sheet.pdf

2. De Groot P., "Principles of interference microscopy for the measurement of surface topography," Advances in Optics and Photonics, 7, 1-65 (2015).

3. Lehmann P., Tereschenko S. and Xie W., "Fundamental aspects of resolution and precision in vertical scanning white-light interferometry," Surf. Topogr.: Metrol. Prop. 4, 0240041 (2016).

4. Chen Z., Taflove A. and Backman V., "Photonic nanojet enhancement of backscattering of light by nanoparticles: a potential novel visible-light ultramicroscopy technique," Opt. Express 12, 1214 (2004).

5. Sun W., Nousiainen T., Muinonen K., Fu Q., Loeb N. G. and Videen G., "Light scattering by Gaussian particles: a solution with finite-difference time domain technique," J. Quantitat. Spectrosc. Radiat. Transfer 79-80, 1083-1090 (2003).

6. White Paper: Blu-ray Disc ${ }^{\mathrm{TM}}$ Format, 1. B Physical Format Specifications for BD-R, 5th Edition, Blu-ray Disc Association (2010).

7. Wu Y., Huang F., Gu D. and Gan F., "Organic materials for recordable blue laser optical storage, " Proc. SPIE 5966, 59661E (2005).

8. Wyant J. C., "White light interferometry, " Proc. SPIE 4737, 98-107 (2002).

9. Harasaki A., Schmit J. and Wyatt J.C., "Improved Vertical-Scanning Interferometry," Appl. Optics 39, $2107-$ 2115 (2000). 